\title{
The Value of I-Scan Image-Enhanced Endoscopy in the Diagnosis of Vocal Cord Leukoplakia
}

\author{
Young Chan Lee ${ }^{1}$, Young-Gyu Eun ${ }^{1}$ and Il-Seok Park ${ }^{2}$ \\ ${ }^{I}$ Department of Otolaryngology-Head and Neck Surgery, School of Medicine, Kyung Hee University, Seoul; and \\ ${ }^{2}$ Department of Otorhinolaryngology-Head and Neck Surgery, Dongtan Sacred Heart Hospital, \\ Hallym University College of Medicine, Chuncheon, Korea
}

\begin{abstract}
$=$ Abstract $=$
Background and Objectives : Detection of vascular abnormalities in vocal cord (VC) leukoplakia is important for the diagnosis of neoplastic change of the mucosa. The aim of this study was to investigate the value of $\mathrm{i}$-scan in the differential diagnosis of VC leukoplakia based on visualization of abnormal vascular features. Material and Methods : Fifty-two patients with leukoplakia were enrolled in the study. Images of their larynx obtained using conventional white light endoscopy and an i-scan-enhanced endoscopy (Pentax DEFINA EPK-3000 Video Processors, with Pentax VNLJ10) were reviewed. The microvascular features of the lesions and vascular changes were analyzed and the results were compared with the histopathologic diagnosis. Results : Among the 52 leukoplakia patients, 7 (13.5\%) patients had squamous hyperplasia, $10(19.3 \%)$ mild dysplasia, 2 (3.8\%) moderate dysplasia, 14 (26.9\%) severe dysplasia, 4 (7.7\%) carcinoma in situ, and $15(28.8 \%)$ invasive squamous cell carcinoma on histopathologic examination. Using i-scan-enhanced endoscopy, abnormal vascular change with neoplastic neoangiogenesis was detected in most cases of malignant VC lesion [severe dysplasia : 9/14 (64.3\%), carcinoma in situ: $2 / 4$ (50.0\%), and invasive squamous cell carcinoma : 11/15 (73.4\%)]. Conclusion : i-scan-enhanced endoscopy is a useful optical technique for the diagnosis of VC leukoplakia. Our results suggest that i-scan may be a promising diagnostic tool in the early detection of laryngeal cancer.
\end{abstract}

KEY WORDS : Leukoplakia $\cdot$ Vocal cord $\cdot$ Carcinoma $\cdot$ Endoscopy.

\section{Introduction}

Vocal cord (VC) leukoplakia is a clinical term for white patches or plaques on the vocal cords, regardless of the histological features. ${ }^{1)}$ The pathologic results of clinically diagnosed VC leukoplakia may be hyperplasia, mild or moderate dysplasia, severe dysplasia, or carcinoma. Furthermore, the degree of atypical hyperplasia is considered to be the most important prognostic factor for evaluating the risk of progression of malignant tumors in VC leukoplakia. ${ }^{2)}$ However, without the histologic examination under general anesthesia of VC leukoplakia, it is difficult to determine the degree of dysplasia. Therefore, predicting the histologic diagnosis with en-

Received: November 5, 2018 / Revised: November 8, 2018

Accepted: November 20, 2018

Address for correspondence: I-Seok Park, MD, PhD, Department of Otorhinolaryngology-Head and Neck Surgery, Dongtan Sacred Heart Hospital, Hallym University College of Medicine, 7 Keunjaebong-gil, Chuncheon 18450, Korea

Tel: (031) 8086-2670, Fax: (031) 8086-2681, E-mail: ispark@hallym.or.kr doscopy may be very important as a diagnostic and followup tool for VC leukoplakia. However, conventional white light endoscopy (WLE) is not optimal in resolution and contrast, which is difficult to distinguish between superficial mucosal cancer and precancerous lesions. Furthermore, for VC leukoplakia, there is an umbrella effect - epithelial keratinization covers underlying vascular changes. ${ }^{3)}$ The narrow band imaging (NBI) system (Olympus Medical Systems, Tokyo, Japan) is a noninvasive optical device that uses reflected light to visualize the superficial structure and enhance the appearance of the vasculature within the mucosal layer. Examination using NBI is greatly related to the morphology of microvessels, which include the intraepithelial papillary capillary loop (IPCL). ${ }^{4)}$ However, narrow band images are much darker than conventional WLE images.

$\mathrm{i}$-scan technology is a newly developed image-enhanced endoscopy technology from PENTAX, Japan. i-scan images are as bright as conventional WLE images and do not need magnifying endoscopy to observe the demarcation of the lesion. ${ }^{5)}$ It has three modes of image enhancement : SE, sur- 
face enhancement (enhancement of the structure through recognition of the edges) ; CE, contrast enhancement (enhancement of depressed areas and differences in structure through colored presentation of low-density areas); and TE, tone enhancement (enhancement tailored to individual organs through modification of the combination of RGB components for each pixel). i-scan technology has been used in gastroenterology practice and has already been shown to improve the accuracy and diagnostic yield by identifying subtle abnormalities during endoscopy. Iacucci et al. have found that $\mathrm{i}$-scan improved the characterization of patchy atrophy of the villi in patients with celiac disease. $\left.{ }^{6}\right)$ This helped in directing and targeting duodenal biopsies and improved the diagnostic yield. Graham and Banks found specialist upper gastrointestinal endoscopists using i-scan could detect dysplasia within a Barrett's segment with $75 \%$ sensitivity.')

We applied the i-scan-enhanced endoscopy (Pentax DEFINA EPK-3000 Video Processors, with Pentax VNLJ10) with stroboscopy in patients with VC leukoplakia. We provided imaging of the lesions obtained using both WLE and iscan-enhanced endoscopy. The aim of this study was to investigate the value of $i$-scan in the differential diagnosis of VC leukoplakia based on abnormal vascular features seen with i-scan.

\section{Materials and Methods}

The study was approved by the institutional ethics committee and performed in accordance with the Declaration of Helsinki.

\section{Subjects}

Between March 2016 and September 2018, 52 untreated patients who were diagnosed with VC leukoplakia clinically and underwent laryngeal microsurgery for tissue biopsy confirmation were enrolled in this retrospective study. The selection criteria for the present study were as follows : 1) the presence of $\mathrm{VC}$ leukoplakia, 2) no previous procedures (e.g., surgery, radiotherapy), and 3) preoperative endoscopy with WL and i-scan. The patients were examined using flexible endoscopy with WLE and i-scan (Pentax DEFINA EPK3000 Video Processors, with Pentax VNLJ10) by one qualified laryngologist. Age, sex, medical history, preoperative laryngoscopy on WL and i-scan-enhanced endoscopy, and pathologic histologic features were analyzed. The examinations were performed first with WL with a wide view to observe the whole lesion and the surrounding mucosa. Then, under i-scan, the same procedure was performed and the vascular features were analyzed in detail and recorded. i-scan consists of three different image algorithms : SE, TE, and CE. The three modes (SE, TE, and CE) are arranged in series ; therefore, it is possible to apply two or more of these modes at one time. Switching of the level or mode of enhancement can be performed on a real-time basis, without any time lag, by pushing a scope button, enabling efficient endoscopic observation. Image analysis of the microvascular features (regular and irregular) of the lesions and vascular change descriptions published by Arens et al. were followed ; type I was defined as longitudinal vascular changes, enlarged and static vessels, meandering, tortuous, or dilated vessels, convoluted vessels, increased number of vessels or branches of vessels "feeding" blood vessels ; type II was defined as perpendicular vascular changes, real vessel loops with a wideangled turning point embedded in a three-dimensional warty structure ; type III was defined as enlarged and symmetric dot-like loops, abnormal IPCLs with narrow-angled turning points ; and type IV was defined as abnormal worm-like vessels with spiral morphology and bizarre course. ${ }^{3)}$

Surgical procedures, such as biopsy, laser surgery, or surgical extirpation under general anesthesia, were performed after the patients provided written consent. The specimens from these patients were obtained from the archives of the Department of Pathology and reviewed by head and neck pathologists to verify the diagnosis and confirm the degree of atypical hyperplasia. Epithelial dysplasia was diagnosed according to the World Health Organization (WHO) classification. $^{8)}$

\section{Results}

Fifty-two patients with leukoplakia were enrolled. The mean age was 62.5 years (range 38 80 years) ; 48 (92.3\%) patients were male and 4 (7.7\%) were female. Among them, 7 (13.5\%) patients had squamous hyperplasia, 10 (19.3\%) mild hyperplasia, 2 (3.8\%) moderate dysplasia, 14 (26.9\%) severe dysplasia, 4 (7.7\%) carcinoma in situ, and 15 (28.8\%) invasive squamous cell carcinoma on histopathologic examination (Table 1). Among the 7 patients with squamous hyperplasia, longitudinal vascular patterns (type I) in the i-scan image were detected in $5(71.4 \%)$ and type II perpendicular vascular changes in $2(28.6 \%)$ patients. Among patients with mild dysplasia, $4 / 10(40.0 \%)$ were identified as having a type I vascular pattern, $4 / 10(40.0 \%)$ as having type II vascular change, and 3/10 (30.0\%) as having type III abnormal IPCLs with narrow-angled turning points. Two leukoplakia lesions found to have moderate dysplasia exhibited type II and type III vascular 
change. Histologic features of severe dysplasia, carcinoma in situ, or invasive squamous cell carcinoma were detected in 33/52 (63.4\%) patients ; i-scan identified neoplastic neoangiogenesis (type IV) in 9/14 (64.3\%), 2/4 (50.0\%), and 11/15 (73.4\%) patients with severe dysplasia, carcinoma in situ, and invasive squamous cell carcinoma, respectively (Table 2).

\section{Discussion}

Early detection of laryngeal carcinoma is always essential because it permits a higher rate of local control and, consequently, a higher survival rate. Therefore, leukoplakia is evaluated using magnifying laryngoscopy and long-term observation is recommended. ${ }^{9)}$ In addition to conventional WLE, many other "biologic endoscopy" methods (e.g., autofluorescence, contact endoscopy with vital tissue staining, and confocal endomicroscopy) have improved the rates of earlier detection of not only epithelial, but also vascular change in vocal cord. ${ }^{10)}$ Among them, narrow band imaging (NBI), which is an endoscopic imaging technique that emphasizes the mucosal microvasculature and identifies vascular alterations by placing narrow bandpass filters in front of a conventional white-light source to obtain tissue illumination at selected, narrow wavelength bands, is increasingly preferred and rec-

Table 1. Demographic \& clinical characteristics of enrolled patients

\begin{tabular}{lc}
\hline \multicolumn{2}{c}{ Variables } \\
\hline Number of enrolled patients & 52 \\
Age & 62.5 years $(38-80$ years $)$ \\
Sex (male/female) & \\
Male & $48(92.3 \%)$ \\
Female & $4(7.7 \%)$ \\
Pathology & \\
Squamous hyperplasia & $7(13.5 \%)$ \\
Mild hyperplasia & $10(19.3 \%)$ \\
Moderate dysplasia & $2(3.8 \%)$ \\
Severe dysplasia & $14(26.9 \%)$ \\
Carcinoma in situ & $4(7.7 \%)$ \\
Invasive Squamous cell carcinoma & $15(28.8 \%)$ \\
\hline
\end{tabular}

ommended for examining in laryngeal lesion. ${ }^{11)}$ i-scan is a newly developed image enhanced endoscopy technology from PENTAX. Although NBI is useful for detecting, diagnosing or identifying the demarcation line and predicting the histological characteristics of cancers with magnifying endoscopy, narrow band images are much darker than conventional white light images, particularly in large luminal diameter regions of the gastrointestinal tract. ${ }^{12)}$ i-scan images are as bright as conventional white-light images, therefore, i-scan is able to observe much larger areas from a distant view than NBI. Moreover, i-scan does not need magnifying endoscopy to observe the demarcation of the lesion.

To our knowledge this is the first study to show the benefit of laryngoscopy combined with i-scan for the diagnosis of vocal cord leukoplakia. This study shows that the use of i-scan image enhancement technology improves the detection of vascular changes, offering the combination of surface enhancement and tone enhancement. Huang et al. showed that microvessel characteristics in the mucosa around the plaque identified using NBI were closely associated with the pathological diagnosis. ${ }^{13)}$ i-scan consists of three types of algorithms : SE, CE, and TE. SE and CE are expected to provide a useful means of screening because they can improve detection of lesions without altering the color tone markedly or reducing the brightness of images. After a lesion is found, TE is applied as a simple means of making changes in the color tone and structure more evident. ${ }^{5)}$ Hoffman et al. demonstrated that HD+ colonoscopy with i -scan functionality detected significantly more patients with colorectal neoplasia compared with standard resolution endoscopy. ${ }^{14)}$ In their study, significantly more neoplastic (adenomatous and cancerous) lesions and more flat adenomas could be detected using high definition endoscopy with surface enhancement. i-scan has proved useful not only in the intestinal mucosa but also in the airway mucosa. In an analysis of 29 patients, vascular abnormalities were scored most frequently in HD + i-scan 2 bronchoscopy than in autofluorescence videobronchoscopy. This study also showed that high definition bronchoscopy with image enhancement techniques may result in better detec-

Table 2. Comparison of i-scan images for vascular feature and histopathologic findings

\begin{tabular}{lllll}
\hline \multirow{2}{*}{ Pathology } & \multicolumn{4}{l}{ Microvascular feature type on i-scan } \\
\cline { 2 - 5 } & \multicolumn{1}{c}{ Type I } & \multicolumn{1}{c}{ Type II } & \multicolumn{1}{c}{ Type III } & 0 \\
\hline Squamous hyperplasia & $5 / 7(71.4 \%)$ & $2 / 7(28.6 \%)$ & 0 & 0 \\
Mild dysplasia & $4 / 10(40.0 \%)$ & $4 / 10(40.0 \%)$ & $3 / 10(30.0 \%)$ & 0 \\
Moderate dysplasia & 0 & $1 / 2(50.0 \%)$ & $1 / 2(50.0 \%)$ & $9 / 14(64.35)$ \\
Severe dysplasia & 0 & 0 & $5 / 14(35.7 \%)$ & $2 / 4(50.0 \%)$ \\
Carcinoma in situ & 0 & 0 & $2 / 4(50.0 \%)$ & $11 / 15(73.4 \%)$ \\
Invasive squamous cell carcinoma & 0 & $1 / 15(6.6 \%)$ & $3 / 15(20.0 \%)$ & \\
\hline
\end{tabular}



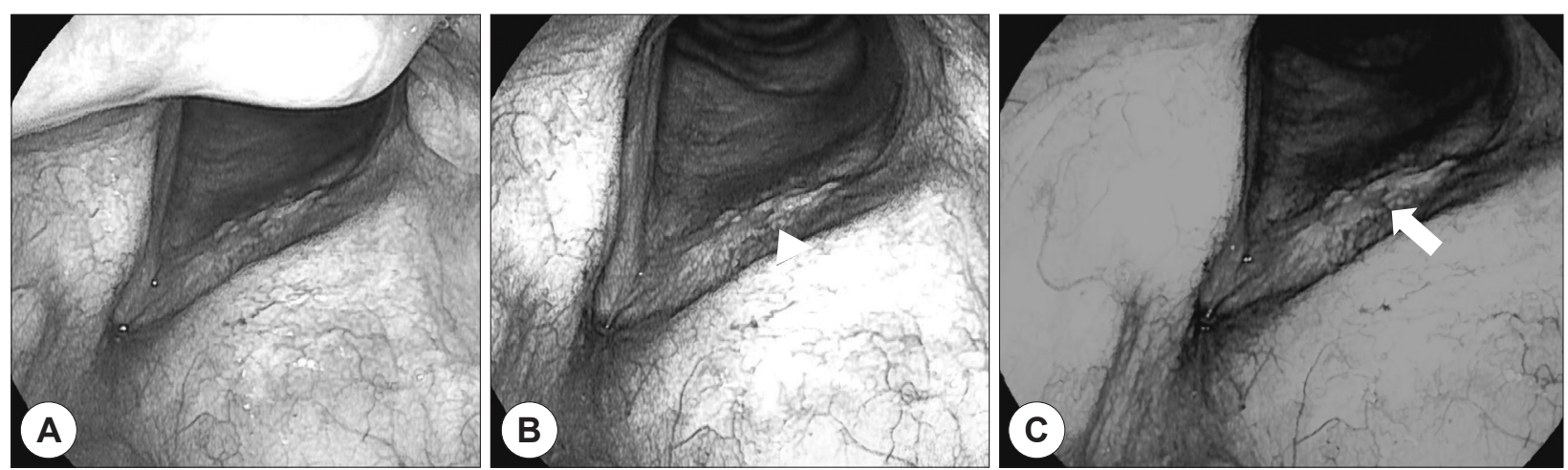

Figure 1. Images using i-scan-enhanced endoscopy. A : i-scan 1 (surface and contrast enhancement). A whitish irregular lesion is seen on the left vocal cord. The lesion could be identified as an irregular area with clear boundaries. B : i-scan image with tone enhancement. i-scan 2 better demarcates the vascular components (arrow head) of a hypervascular lesion in leukoplakia. C : i-scan 3 (surface and tone enhancement). i-scan 3 characterizes the abnormal vascular change with worm-like features (arrow).

tion of subtle vascular abnormalities in the airways. ${ }^{15)}$

In our study, type IV abnormal worm-like vessels with spiral morphology and bizarre course were detected mainly in malignant lesions, such as high-grade dysplasia, carcinoma in situ, and invasive cancer (Fig. 1). Perpendicular vascular networks are characterized by the development of IPCLs. The main target of analysis in i-scan techniques is neoangiogenesis, and observation of thick dark spots within a welldemarcated brownish area with proliferation of dilated and abnormal IPCLs is generally considered a hallmark of epithelial precancerous or neoplastic evolution. In most leukoplakic lesions proven to be malignant, abnormal IPCLs were detected on i-scan images. The growth of epithelial tumors leads to escalated disorderliness of the vascular microarchitecture, which is observed in practice as changes in the IPCL arrangement, diameter, and shape, and as a loss of regularity. ${ }^{3)}$ Stanikova et al. demonstrated that 22/26 cases that had malignant IPCLs on NBI were confirmed to be carcinoma in situ or invasive squamous cell carcinoma. Our results are in line with these findings.

Detection, demarcation, and characterization are the most important first steps during endoscopic examinations in patients with laryngeal cancer. i-scan SE highlights VC mucosal surface structures that can be used for the detection of epithelial changes. Abnormalities are easier to detect. i-scan TE accentuates mucosal structures and vascular patterns, improving the determination of lesion margins. These details are helpful in demarcating and characterizing VC leukoplakia and degree of dysplasia. Neoplastic and inflammatory vessel and tissue patterns may be differentiated from nonneoplastic patterns. i-scan 3 (SE and TE) further enhances the visibility of the vessel patterns and helps in the localization of safe biopsy sites.

This study has some limitations. First, we performed a ret- rospective image review, not prospective studies. There is a possibility that the description of the vascular pattern may be different depending on the part of the image taken. Second, in the present study we did not analyze surface mucosal change or epithelial abnormalities, which are important variables in endoscopic diagnosis. In future, prospective studies should be conducted to address these limitations and to validate the value of i-scan by comparing its efficacy with that of NBI.

\section{Conclusion}

Laryngoscopy with an i-scan-enhanced technique is useful in detecting subtle vascular patterns in leukoplakia. These vascular abnormalities could be significantly related to malignant histopathologic findings. Further studies for validating this technique for the diagnosis of laryngeal cancer are needed.

\section{REFERENCES}

1) Frangež I, Gale N, Luzar B. The interpretation of leukoplakia in laryngeal pathology. Acta Oto-Laryngologica 1997;117(sup527): 142-4.

2) Isenberg JS, Crozier DL, Dailey SH. Institutional and comprehensive review of laryngeal leukoplakia. Annals of Otology, Rhinology \& Laryngology 2008;117(1):74-9.

3) Arens C, Piazza C, Andrea M, Dikkers FG, Gi RETP, Voigt-Zimmermann S, et al. Proposal for a descriptive guideline of vascular changes in lesions of the vocal folds by the committee on endoscopic laryngeal imaging of the European Laryngological Society. European Archives of Oto-Rhino-Laryngology 2016;273(5):1207-14.

4) Ni X, He S, Xu Z, Gao L, Lu N, Yuan Z, et al. Endoscopic diagnosis of laryngeal cancer and precancerous lesions by narrow band imaging. The Journal of Laryngology \& Otology 2011;125(3):288-96.

5) Kodashima S, Fujishiro M. Novel image-enhanced endoscopy with i-scan technology. World Journal of Gastroenterology: WJG 2010;16(9):1043-9.

6) Iacucci M, Poon T, Gui XS, Ghosh S. High definition i-SCAN endoscopy with water immersion technique accurately reflects histological severity of celiac disease. Endoscopy International Open 
2016;4(5):E540.

7) Graham DG, Banks MR. Advances in upper gastrointestinal endoscopy. F1000Research 2015;4.

8) Barnes L, Eveson JW, Reichart P, Sidransky D. Pathology and genetics of head and neck tumours: IARC;2005.

9) Korean Society of Thyroid-Head and Neck Surgery Guideline Task Force, Ahn SH, Hong HJ, Kwon SY, Kwon KH, Roh JL, et al. Guidelines for the surgical management of laryngeal cancer: Korean Society of Thyroid-Head and Neck Surgery. Clin Exp Otorhinolaryngol 2017;10(1):1-43.

10) Stevens SM, Johnson EA, Pfau PR, Dailey SH. Visual evaluation of the larynx and hypopharynx during esophagogastroduodenoscopy: a safety and feasibility study. Surgical endoscopy 2015;29(5):1209-15.

11) Kuznetsov K, Lambert R, Rey J-F. Narrow-band imaging: potential and limitations. Endoscopy 2006;38(01):76-81.

12) Yao K, Anagnostopoulos G, Ragunath K. Magnifying endoscopy for diagnosing and delineating early gastric cancer. Endoscopy 2009;41(05):462-7.

13) Huang F, Yu J, Zhang F, He C, Li S, Shao J. The usefulness of narrow-band imaging for the diagnosis and treatment of vocal fold leukoplakia. Acta Oto-Laryngologica 2017;137(9):1002-6.

14) Hoffman A, Sar F, Goetz M, Tresch A, Mudter J, Biesterfeld S, et al. High definition colonoscopy combined with $i$-Scan is superior in the detection of colorectal neoplasias compared with standard video colonoscopy: a prospective randomized controlled trial. Endoscopy 2010;42(10):827-33.

15) van der Heijden EH, Hoefsloot W, van Hees HW, Schuurbiers OC. High definition bronchoscopy: a randomized exploratory study of diagnostic value compared to standard white light bronchoscopy and autofluorescence bronchoscopy. Respiratory Research 2015; 16(1):33. 